\title{
Hemorragias de la segunda mitad de la Gestación.
}

\section{CORRELATO DE LA SOCIEDAD DE OBSTETRICIA Y GINECOLOGIA DE BOLIVAR}

Revisión de 3 años en los Hospitales: "UNIVERSITARIO DE CARTAGENA" y "MATERNIDAD RAFAEL CALVO C."

Doctores: BENJAMIN BLANCO MARTINEZ, M.D.*
DONALDO PEREZ PEREZ, M.D.**

JORGE CABRALES A., M.D.***

FERNANDO MENESES, M.D.***

\section{INTRODUCCION}

Las hemorragias de la segunda mitad de la gestación, para muchos tratadistas y escuelas, son denominadas del tercer trimestre. En la Clínica de Maternidad "RAFAEL CALVO C." y en el HOSPITAL UNIVERSITARIO DE CARTAGENA, las hemorragias del embarazo, eminentemente obstétricas, se dividen en hemorragias de la primera y de la segunda mitad de la gestación. Entre las de la segunda mitad, se encuentran: Placenta Previa, Desprendimiento Prematuro de Placenta Normo Inserta (D.P. P.N.I.) y Ruptura Uterina. El cuadro de ruptura del seno marginal, se incluye en el de D.P.P.N.I. (5). Es preciso diferenciar estas hemorragias eminentemente obstétricas de las que pudieramos llamar ginecológicas; como son las producidas por Cervicitis, $\mathrm{Ca}$. de Cervix, Várices (vaginal, vulvar o rectal), Condilomas, Polípos, Desgarros de fondos de sacos de la vagina, Vulvovaginitis sangrante (causadas por Tricomonas, Candida

* PROFESOR ASOCIADO, del Departamento de OBSTETRICIA-GINECOLOGIA, F Acultad de Medicina-Universidad de Cartagena.

** PROFESOR ASISTENTE, del Departamento de OBSTETRICIA-GINECOLOGIA, Facultad de Medicina-Universidad de Cartagena.

*** RESIDENTES III, del Departamento de OBSTETRICIA-GINECOLOGIA, Facultad de Medicina-Universidad de Cartagena.
Albicans o Bacterias), lesiones vulvovaginales por venéreas, etc. Estas entidades que nada tienen que ver con el embarazo, a menudo se presentan con él y, no raras veces, confunden al médico general en su diagnóstico de hemorragias de la segunda mitad de la gestación.

Para nuestra revisión se tomará el criterio más moderno, el de Hemorragias de la segunda mitad de la gestación, $(2,5)$.

\section{PLACENTA PREVIA}

La Placenta Previa se divide en: TOTAL, PARCIAL y DE INSERCION BAJA, $(4,5)$. En la Placenta Previa Total el orificio interno está totalmente cubierto por la placenta. En la Placenta Previa Parcial el orificio interno está parcialmente cubierto por la placenta. En la Placenta Previa de Inserción Baja, la placenta solo llega al segmento inferior o al margen del orificio interno, pero no lo cubre. El borde de la placenta puede ser palpado por el dedo explorador introducido a través del cuello. Existe una variedad de esta anomalía, en la cual el borde de la placenta invade el margen del orificio interno en una considerable extensión, sin extenderse más allá de él; es lo que se llama, a veces Placenta Previa Marginal, (5).

El grado de la Placenta Previa, depende, en no pequeña medida, de la dilatación cervical existente en el momento de la exploración.

\section{Frecuencia:}

La Placenta Previa es una complicación bastante grave, pero poco frecuente, que se observa una vez en cada 200 partos. Las estadísticas contradictorias sobre la incidencia de los distintos grados de la Placenta Previa reflejan la falta de precisión en sus definiciones. En 304 casos de esta complicación estudiados en el JOHN HOPKINS 
HOSPITAL por Gutiérrez-Yepez y Eastman, el tipo de placenta total fue encontrado en un $23 \%$ de casos, el parcial en $29 \%$ y la placenta de inserción baja en un $48 \%$, (5).

\section{Etiología:}

Poco se conoce acerca de la causa de la Placenta Previa. Sin embargo, la multiparidad y la edad avanzada parecen favorecer su aparición. Es casi el doble más frecuente en multíparas que en primigrávidas. Las mujeres de más de 35 años presentan Placenta Previa con una frecuencia de 3 y media veces mayor quelas menores de 25 años. Cuando se excluye el número de partos y se consideran sólamente a las primigrávidas, el aumento de la placenta previa con la edad es, sin embargo, sorprendente, (5).

Los síntomas y signos más comunes de la Placenta Previa, son: sangrado indoloro que cede espontáneamente, presentaciones altas y/o viciosas, la frecuencia cardiaca fetal ( F. C.F.) generalmente está normal, o con leves variaciones. Cuando hay dolor se debe a las contracciones uterinas y por consiguiente sería intermitente. En algunos casos la hemorragia es de cierta magnitud que lleva a la paciente a presentar los signos de hipovolemia y/o shock. Cuando ésto ocurre el feto está en sufrimiento y puede morir.

\section{DESPRENDIMIENTO PREMATURO DE PLACENTA NORMO INSERTA} (D.P.P.N.I.)

Se denomina D.P.P.N.I. al proceso de separación que sufre la placenta norrnalmente implantada, de su conexión uterina, entre las 20 semanas y el término del embarazo, pero antes de la expulsión fetal. Pueden aparecer todos los grados de D.P.P.IN.I. desde un área de pocos milímetros de diámetro hasta la placenta entera, con síntomas y signos de variable intensidad. La gran variabilidad en las manifestaciones clínicas y patológicas del desprendimiento prematuro de la placenta normo-inserta, hacen incierta la clasificación, (4.5).

Frecuencia:

Los límites de incidencia descritos, en- tre 1 por cada 55 partos a 1 por cada 150 , dependen de los criterios de diagnóstico utilizados, (5).

\section{Etiología:}

La causa principal del D.P.P.N.I. es desconocida, pero se han evocado algunos factores etiológicos: traumatismo, cortedad del cordón umbilical, hipertensión crónica, toxemia, presión del útero aumentando de volumen sobre la vena cava inferior y déficit dietético.

Los síntomas y signos más comunes, son: Hemorragia con dolor abdominal, hiperesteșia e hipertonía uterina, sufrimiento o muerte fetal, signos de hipovolemia hasta el shock, evidencias anatómicas placentarias en forma de coágulos y zonas de depresión (lisis) sobre la superficie materna. El líquido amniótico puede estar teñido de sangre, $(4,5)$.

\section{RUPTURA UTERINA}

El útero puede romperse durante el embarazo o más frecuentemente, durante el parto.

Las rupturas del útero durante el parto se dividen en tres clases: Espontáneas, Traumáticas y Dinámicas, (4).

Las causas predisponentes de rupturas espontáneas, incluyen todos los factores mecánicos que obstruyen el descenso del feto a través del canal del parto (distocias mecánicas). Además, los factores que debilitan la pared uterina también pueden causar rupturas, incluyendo cicatrices de miomectomías y cesáreas, multiparidad y embarazo interesticial.

Las rupturas del segmento uterino suelen ser longitudinales y oblicuas; las del cuerpo $\mathrm{y}$ fondo suelen ser transversales.

Las contracciones normales del útero, sólamente pueden causar rupturas, si la pared está lesionada. En presencia de obstrucciones mecánicas incluso el músculo normal puede romperse con contracciones intensas. 
Deben tenerse presente los efectos peligrosos de la estimulación excesiva con agentes útero-estimulantes como la Oxitocina.

Las causas más frecentes de la ruptura traumática son: versión y extracción podálica, parto con obstrucción, no tratado, forceps a través de un cuello no completamente dilatado y traumas externos.

Las multíparas son afectadas de 8 a 9 veces más frecuentemente que las nulíparas y el peligro aumenta con la paridad. Las mujeres obesas parecen tener predisposición especial a la ruptura del útero, (4).

La frecuencia es de 1 por 1.300 , o sea 7.7 por 10.000 partos, (4)

Anatomopatológicamente las rupturas suelen ser divididas en: completas, cuando el comprometimiento llega al peritoneo visceral e incompletas, en la cual el músculo es desgarrado, pero el peritoneo sigue integro, (4).

\section{MATERIAL Y METODOS}

El trabajo se realizó en la CLINICA DE MATERNIDAD "RAFAEL CALVO C." y en el HOSPITAL UNIVERSITARIO DE CARTAGENA, donde se atienden pacientes de clase socio-económica baja. En estas instituciones funciona el departamento de OBSTETRICIA y GINECOLOGIA de la FACULTAD DE MEDICINA de la UNIVERSIDAD DE CARTAGENA.

El material se extrajo de 25.286 Historias de pacientes admitidas durante los años $1976 / 77 / 78$, de las cuales 21.344 correspondieron a embarazos mayores de $20 \mathrm{se}$ manas y 3.942 a embarazos menores de 20 semanas. Las Historias correspondientes a pacientes con hemorragias de la segunda mitad de la gestación (186) fueron clasificadas así: Placenta Previa, 91 casos; D.P.P. N.I., 91 casos y Ruptura Uterina, 4 casos.

Los datos obtenidos fueron tabulados según los parámetros siguientes:

1. Grupos por entidades y años
2. Edad de las pacientes

3. Paridad.

4. Edad gestacional.

5. Motivo de consulta.

6. Síntomas, signos y antecedentes maternos.

7. Tipo de parto

8. Patología asociada.

9. Complicaciones.

10. Tipo de tratamiento

11. Morbi-mortalidad materna y fetal.

12. Grupo sanguíneo y Factor Rh.

\section{RESULTADOS}

El total de casos registrados, de Hemorragias de la segunda mitad de la gestación. durante los 3 años considerados, fué de 186. Al relacionar estos casos con el total de embarazos mayores de 20 semanas (21. 344 ), anotamos que aproximadamente 9 de cada mil embarazadas en este período de gestación padecen de hemorragia.

Considerando los casos año por año, la incidencia de la hemorragias durante el período del embarazo considerado, fluctúa entre 7.8 por mil para 1976 y 10.4 por mil para 1978.

Al clasificar estas hemorragias según entidades, resultaron para los 3 años 91 pacientes con Placenta Previa, el mismo número con D.P.P.N.I. y solo 4 Rupturas Uterinas. La distribución del número de casos y su incidencia para las dos primeras entidades, en los años 1976-1977, son muy similares, aumentando un poco para 1978. En cuanto a la importancia relativa de las entidades en cada año, la diferencia entre placenta previa y D.P.P.N.I. para el primer año no es muy marcada, gana importancia la Placenta Previa en el año 1977; pero para el año siguiente el D.P.P.N.I. obtiene la primacía. La Ruptura Uterina es poco frecuente; sin embargo, como se verá más adelante, esta entidad es importante por su alta mortalidad fetal.

En cuanto a la edad se refiere, no existe diferencia importante entre las pacientes con Placenta Previa y con D.P.P.N.I. Para la primera, la edad promedio es de 29.1 años, 
para la otra es de 29.6 años. Sin embargo, las pacientes menores de 30 años de edad, en la Placenta Previa son de $59.4 \%$, mientras que en el D.P.P.N.I. es de $46.2 \%$.

Se aprecia que el D.P.P.N.I. es 3 veces más frecuente en las nulíparas que la Placenta Previa. Para las de paridad de 1 a 3 es más frecuente la Placenta Previa $(49.4 \%$ o) que el D.P.P.N.I. $(37.3 \%)$. Las pacientes con Placenta previa tiene un promedio de 3.8 partos. Las de D.P.P.N.I., alcanzan 4 partos.

Podemos ver, que tanto la Placenta Previa como el D.P.P.N.I., son relativamente poco frecuentes entre las 20 y 28 semanas de embarazo. Se presentan con marcada frecuencia desde las 34 y más semanas de embarazo. El promedio de semanas de embarazo en que se presentan las dos entidades, es de 36. Los casos de Rupturas Uterinas, todos, fueron en embarazos a término.

El motivo de consulta predominante, en la Placenta Previa y en el D.P.P.N.I. fue el sangrado indoloro y el sangrado con dolor. En la Placenta Previa predominó el sangrado indoloro $(81.3 \%$ o), mientras que en el D.P.P.N.I. predominó el sangrado con dolor $(59.3 \%$ o). El dolor fué el síntoma que predominó en la Ruptura Uterina.

Se aprecia que solo el $37 \%$ de los casos presentaron síntomas, signos o antecedentes maternos asociados. Entre éstos la. hipertonía uterina con $42 \%$ exclusiva del D.P.P. N.I. La cefalea predominó en la Placenta Previa. En la Ruptura Uterina se halló situación transversa del feto $(1.4 \%)$, cesárea (14\%).

Anotamos que el tipo de parto predominante fue la Cesárea segmentaria transperitoneal, con $84 . \%$. Este es seguido por el parto vaginal espontáneo con $10.7 \%$. El parto vaginal conducido, solo fue en $3.2 \%$, y únicamente en pacientes con D.P.P.N.I. La patología asociada estuve presente en $22.5 \%$ de los casos. De este porcentaje corresponde a la toxemia gravídica $35.5 \%$, a la enfermedad vascular hipertensiva crónica $9.5 \%$ a la infección urinaria y a la vulvovaginitis $14.5 \%$, a la cervicitis crónica $12 \%$ y en menor porcentaje otras entidades.

Las complicaciones maternas se presentaron en $10.2 \%$ de todos los casos; siendo mucho más común en el D.P.P.N.I. (79\%). Sobresalen el Utero de Couvelaire (42.2\%) y la coagulación intravascular diseminada $-\mathrm{C}$. I.D.- $(13.5 \%)$.

Todos los casos se trataron con líquidos y electrolitos como medidas generales, además con cortoides y sangre total 48 casos, (25\%). El uso de Heparina fue exclusivo para el D.P.P.N.I. en 25 casos (27.5\%). De éstos, 21 (84\%) evolucionarion satisfactoriamente y 4 casos $(16 \%)$ murieron. El ácido epsilon amino-caproico se utilizó en un caso $(0.6 \%)$.

Entre otros tipos de cirugía realizados, la Salpingectomía parcial bilateral predominó con 42 casos $(22.5 \%)$;

La mortalidad fetal fue de 57 casos (30.6\%). De esta, corresponde a Placenta Previa $15.8 \%$, al D.P.P.N.I. $48.4 \%$ y a la Ruptura -Uterina 7\% . Analizadas separadamente estas entidades, según número de casos, corresponde a la Placenta Previa 9.9\%, al D.P.P.N.I. $48.4 \%$ y a la Ruptura Uterina $100 \%$. La mortalidad materna fue exclusiva del D,P,P, N.I., con un porcentaje específico para éste de $8.8 \%$ y general de $4.3 \%$.

En el momento del ingreso hospitalario, de los 57 mortinatos, se halló que 35 $(61.4 \%)$ estaban muertos, $19(33.3 \%)$ tenían sufrimiento, uno $1.8 \%$ ) estaban nor mal y $2(3.5 \%)$ sin datos.

En nuestra serie se hemoclasificaron 124 casos $(66.7 \mathrm{O} / \mathrm{o})$. De este porcentaje corresponde a Placenta Previa $42 \%$ o, al D.P.P.N.I. $55.6 \%$ y a la Ruptura Uterina 2.4 o/o. Analizadas separadamente las 3 entidades, según número de casos, se halla para D.P.P. N.I. y para Ruptura Uterina, el mismo porcentaje $(75 \% / 0)$ y para Placenta Previa $57 \%$. El análisis de los grupos separadamente, revela: Cero positivo $\left(0_{+}\right) 59.6 \%$; 
$(\mathrm{A}+) 17 \%$ o; $\left(\mathrm{B}_{+}\right) 12 \%$ y $8^{\circ} \%$ o para los grupos $\mathrm{O}-\mathrm{A}-\mathrm{B}-\mathrm{y} \mathrm{AB}$ negativos. De este $8 \%$ negativo corresponde a la Placenta Previa $6.4 \mathrm{o} / \mathrm{o}$ y al D.P.P.N.I. $1.6 \mathrm{o} / \mathrm{o}$.

Las complicaciones presentes en las pacientes que murieron, fueron: coagulación intravascular diseminada -C.I.D.- $50 \%$, insuficiencia renal aguda -I.R.A.- $37.5 \%$ y eclampsia $12.5 \%$. En el $100 \%$ o de estos casos se usó sangre total y corticoides. La Heparina se usó en el 50 \% /o de ellos.

\section{COMENTARIOS}

La revisión nuestra obtuvo un total de 186 casos y una frecuencia de 9 por cada mil embarazadas. Considerando los casos año por año, la incidencia fluctúa entre 7.8 por mil para 1976 y 10.4 por mil para 1978. El total de casos para Placenta Previa y D.P.P.N.I. fue el mismo (91). El total de Rupturas solo llegó a 4 casos.

La edad promedio, para placenta previa y D.P.P.N.I. fue de 29 años. Sin embargo, las pacientes menores de 30 años, en la Placenta Previa son $59.4 \%$ y en el D.P.P.I. $46.2 \%$ o.

En las nulíparas el D. P.P.N.I. es 3 veces más frecuente que la Placenta Previa. Las pacientes con Placenta Previa tienen un promedio de 3.8 partos. Las de.D.P.P.N.I. de 4 partos.

En embarazos mayores de 34 semanas, la Placenta Previa y el D.P.P.N.I., se presentaron con marcada frecuencia. El promedio de semanas de gestación, para ambas entidades fue de 36 . En la Ruptura Uterina, todos los embarazos estaban a término.

El motivo de consulta, predominante, en la Placenta Previa fue el sangrado indoloro $(81.3 \%$ o). El sangrado con dolor predominó en el D.P.P.N.I. $(59.3 \% / 0)$ y el dolor en la Ruptura Uterina $(75 \%$ o).

La hipertonía estuvo presente en el D.P.P. N.I. con $42 \%$. La situación transversa del feto, la cesárea anterior y el manejo por em- pírica, se asociaron con la Ruptura Uterina en $75 \%$ de estos casos.

La toxemia gravídica se asoció a estas hemorragias de la segunda mitad de la gestación, en $8 \%$. La enfermedad vascular hipertensiva crónica en $2.2 \mathrm{o} / \mathrm{o}$. Las complicaciones maternas se presentaron en $10.2 \%$ de todos los casos; siendo mucho más común en el D.P.P.N.I., 79 \%. Sobresalen el Utero de Couvelaire $(42.2 \%$ y la C.I.D. (13.5 o/o).

El tipo de parto que predominó fue el intervenido por cesárea segmentaria transperitoneal, 84.9\% $\%$. Este es seguido luego por el parto vaginal espontáneo con $10.7 \%$.

En el manejo de estos pacientes, destacamos que el 60 \% de ellos se trataron sólamente, con líquidos y electrolitos, además de otras medidas generales y especiales; el $25 \%$ recibió corticoides y sangre total. El uso de Heparina fue exclusivo para el D.P. P.N.I. en 25 casos $(27.5 \%$ o). De éstos, 21 (84 o/o) evolucionaron satisfactoriamente y 4 casos $(16 \% / 0)$ murieron.

La salpingectomía Parcial Bilateral se hizo en 42 casos $(22.6 \mathrm{o} / \mathrm{o})$, la Histerectomía Subtotal en 13 casos $(7 \mathrm{o} / \mathrm{o})$ y la Total en 2 casos $(1 \%)$.

La mortalidad fetal en nuestra serie fue de 57 casos (30.6 \%). De éste, corresponde a la Placenta Previa 4.9 o/o; al D.P.P.N.I., $23.5 \%$ y a la Ruptura Uterina, $2.2 \%$. Analizada esta mortalidad, separadamente, para cada entidad, según número de casos, corresponde a la Placenta Previa, 9.9 o/o; al D.P.P.N.I., 48.4 \% y a la Ruptura Uterina, 100 \% . Llama la atención esta mortalidad, que sigue siendo alta y muy especialmente, en la Ruptura Uterina $\left(100^{\circ} /\right.$ o) y el D.P.P. N.I.; $48.4^{\circ} /$ o.

El $61.4 \%$ de los fetos que nacieron ya estaban muertos y en el momento de su ingreso, $33.3 \%$ tenían.sufrimiento $\mathrm{y}$, solo 1.8 $\%$, estaban normales.

La mortalidad materna, fue exclusiva del D.P.P.N.I., con 8 casos $(8.8 \%$ o). Para el nú- 
mero total de casos (186), esta rnortalidad corresponde a $4.3 \mathrm{o} / \mathrm{o}$.

Como causas eficientes de la rortalidad materna, señalamos la C.I.D. en $50 \%$ o, la I.R.A., en $37.5 \mathrm{o} / \mathrm{o}$ y a la Eclampsia en $12.5 \%$. En el $100 \%$ de estos (casos) se usó sangre total y corticoides, además se uso Heparina en el $50 \%$ o de ellos.

Llama la atención, que de 52 casos $57 \%$ o hemoclasificados en la placenta previa, 8 casos $(15.4 \mathrm{o} / \mathrm{o})$ son de factor $\mathrm{Rh}$ negativo.

\section{CUADRO No. 1}

\section{HEMORRAGIAS DELA SEGUNDA MITAD DE LA GESTACION GRUPOS DE PACIENTES POR ENTIDADES Y AÑOS}

ENTIDADES

\begin{tabular}{|lcccccccc} 
& \multicolumn{2}{c}{1976} & \multicolumn{2}{c}{1977} & \multicolumn{2}{c}{1978} & \multicolumn{2}{c}{ TOTAL } \\
& No. & $\% / \mathrm{o}$ & No. & $\%$ & No. & $\%$ & No. & $\%$ \\
PLACENTA PREVIA & 27 & 51 & 29 & 52,7 & 35 & 44,9 & 91 & 48,8 \\
D.P.P.N.I. & 26 & 49 & 24 & 43,6 & 41 & 52,5 & 91 & 48,8 \\
RUPTURA UTERINA & $(0)$ & & 2 & 3,7 & 2 & 2,6 & 4 & 2,4 \\
TOTAL & 53 & $100 \%$ & 55 & $100 \%$ & 78 & $100 \%$ & 186 & $100 \%$ \\
& $(28 \%)$ & & $(29 \%)$ & & $(43 \%)$ & & & \\
\hline
\end{tabular}

CUADRO No. 2

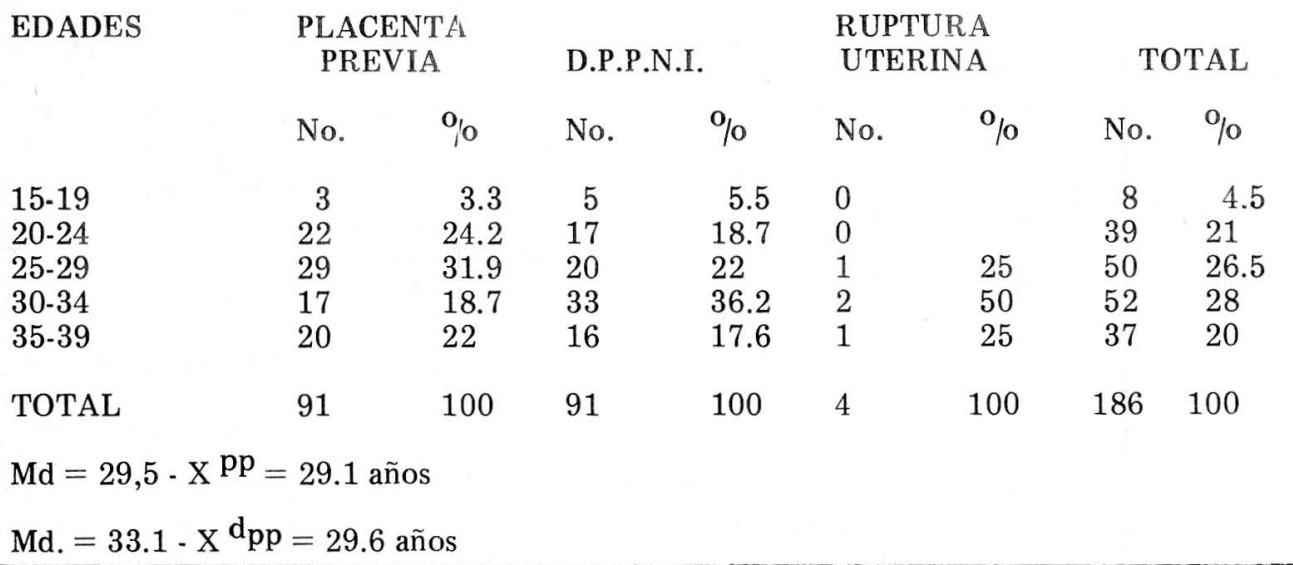




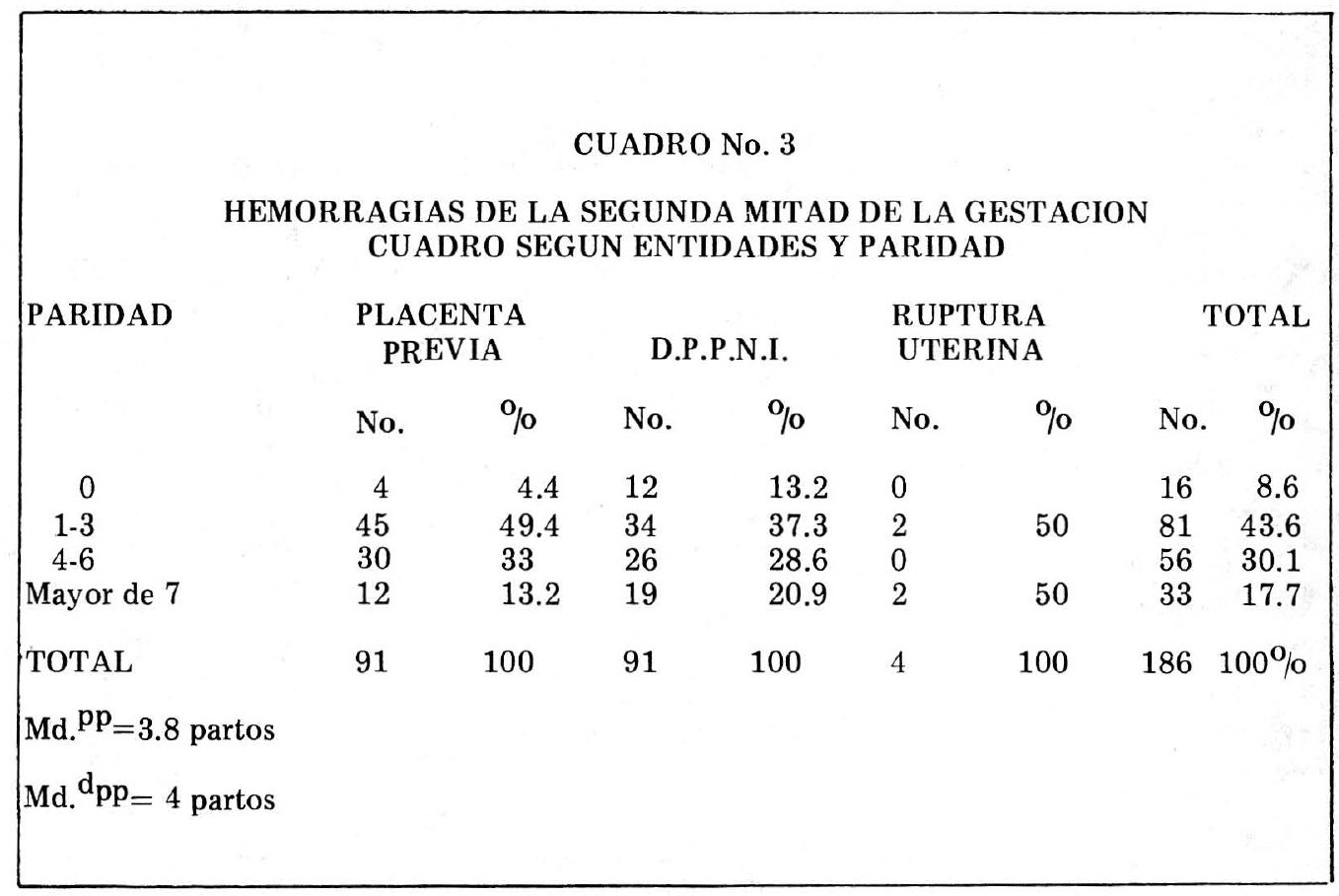

CUADRO No. 4

HEMORRAGIAS DE LA SEGUNDA MITAD DE LA GESTACION
CUADRO SEGUN EDAD GESTACIONAL

SEMANAS DE

EMBARAZO

\section{PLACENTA}

PREVIA
RUPTURA
D.P.P.N.I. UTERINA
TOTAL
20-24

25-28

29. 33

34-36

$37-40$

TOTAL

No.

$\%$

No.

$\%$

No.

$\%$

No. $\%$

$\begin{array}{rrrrr}1 & 1.1 & 4 & 4.4 & 0 \\ 2 & 2.2 & 5 & 5.5 & 0 \\ 24 & 26.4 & 17 & 18.7 & 0 \\ 30 & 33 & 28 & 30.8 & 0 \\ 34 & 37.3 & 37 & 40.6 & 4\end{array}$

$91 \quad 100$

91

100

0
0
0
0
4
4

$\begin{array}{lrc} & 5 & 2.7 \\ & 7 & 3.8 \\ & 41 & 22 \\ 58 & 58 & 31.1 \\ 100 & 75 & 40.4 \\ & & \\ 100 & 186 & 100 \%\end{array}$

Md. ${ }^{p p}=36$ semanas de gestación

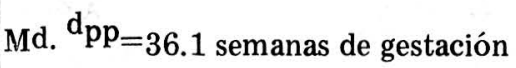


CUADRO No. 5

HEMORRAGIAS DE LA SEGUNDA MITAD DE LA GESTACION MOTIVO DE CONSULTA EN LAS TRES ENTIDADES

MOTIVO DE

PLACENTA

PREVIA

D.P.P.N.I.

RUPTURA

UTERINA

TOTAL

No. $\%$ No. $\%$ No. $\%$ No. $\%$

Sangrado Vaginal 74

$81.3 \quad 17$

18.70

$91 \quad 48.9$

Sangrado vag. ${ }^{+}$dolor 15

$1.6 .5 \quad 54$

$59.3 \quad 0$

$69 \quad 37.1$

abdominal

Dolor abdominal

Convulsiones

$2.2 \quad 17$

$18.7 \quad 3$

75

22

11.8

Fiebre $^{+}$liquido por

3

3.30

$3 \quad 1.6$

0

1

$25 \quad 1$

0.6 genitales

TOTAL

91

100

91

100

4

$100 \quad 186$ $100 \%$

\section{CUADRO No. 6}

\section{HEMORRAGIAS DE I_A SEGUNDA MITAD DE LA GESTACION} SINTOMAS SIGNOS Y ANTECEDENTES MATERNOS ASOCIADOS

SINTOMAS SIGNOS

Y ANTECEDENTES

PLACENTA

PREVIA
RUPTURA

D.P.P.N.I. UTERINA
TOTAL

No. $\%$

Hipertonia uterina

0

29

0

29

42

Presión arterial alta

Hipotensión

Vomitos

Mareos

Cefaleas

Visión borrosa

Disuria

Situación transversa

Cesarea anterior

Manejo por empirica

TOTAL
1

0

3

0

9

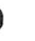

3

7

2

1

0

0

0

14

6

1

1

2

1

1

0

0

0

51

0
1
0
0
0
0
0
1
1
1




\begin{tabular}{|c|c|c|c|c|c|c|c|c|c|}
\hline \multicolumn{10}{|c|}{ CUADRO No. 7} \\
\hline \multicolumn{10}{|c|}{$\begin{array}{l}\text { HEMORRAGIAS DE LA SEGUNDA MITAD DELA GESTACION } \\
\text { CUADRO COMPARATIVO SEGUN TIPO DE PARTO }\end{array}$} \\
\hline \multirow{2}{*}{\multicolumn{2}{|c|}{ TIPO DE PARTO }} & \multicolumn{2}{|c|}{$\begin{array}{l}\text { PLACENTA } \\
\text { PREVIA }\end{array}$} & \multicolumn{2}{|c|}{ D.P.P.N.I. } & \multicolumn{2}{|c|}{$\begin{array}{r}\text { RUPTURA } \\
\text { UTERINA }\end{array}$} & \multicolumn{2}{|c|}{ TOTAL } \\
\hline & & No. & $\%$ & No. & $\%$ & No. & $\%$ & No. & $\%$ \\
\hline \multirow{2}{*}{ VAGINAL } & Espontaneo & 8 & 8.8 & 11 & 12.1 & 1 & 25 & 20 & 10.7 \\
\hline & Conducido & 0 & & 6 & 6.6 & 0 & & 6 & 3.2 \\
\hline \multirow{3}{*}{ CESAREA } & Segmentaria & 82 & 90.1 & 74 & 81.3 & 2 & 50 & 158 & 84.9 \\
\hline & Segmentocorporal & 1 & 1.1 & 0 & & 0 & & 1 & 0.6 \\
\hline & Corporal & 0 & & 0 & & 1 & 25 & 1 & 0.6 \\
\hline TOTAL & & 91 & 100 & 91 & 100 & 4 & 100 & 186 & $100 \%$ \\
\hline
\end{tabular}

CUADRO No. 8

HEMORRAGIAS DELA SEGUNDA MITAD DE LA GESTACION CUADRO COMPARATIVO SEGUN PATOLOGIA ASOCIADA

PATOLOGIA ASOCIADA PLACENTA PREVIA $\begin{array}{ccc}\text { D.P.P.N.I. } & \text { RUPTURA } & \text { TOTAL } \\ \text { UTERINA } & \text { No. } \%\end{array}$

Preeclampsia leve

Preeclampsia severa 1

Eclampsia

Hipertensión arterial

Infeccíón urinaria

Vulvo-vaginitis

Cervicitis cronica

Ruptura prematura de membranas

Amnionitis

TOTAL

$\begin{array}{rr}1 & \mathbf{1} \\ 1 & 8 \\ 0 & 4 \\ 0 & 4 \\ 4 & 2 \\ 6 & 0 \\ 4 & 1 \\ 2 & 1 \\ 1 & 0 \\ 19 & 21\end{array}$

$\begin{array}{rrr}0 & 2 & 4.7 \\ 0 & 9 & 21.3 \\ 0 & 4 & 9.5 \\ 0 & 4 & 9.5 \\ 0 & 6 & 14.4 \\ 0 & 6 & 14.4 \\ 0 & 5 & 12 \\ 1 & 4 & 9.5 \\ 1 & 2 & 4.7 \\ 2 & 42 & 100 \%\end{array}$


CUADRO No. 9

HEMORRAGIAS DE LA SEGUNDA MITAD DE LA GESTACION COMPLICACIONES MATERNAS

\section{TIPO DE COMPLICACION}

C:I-D.

Utero de Couvelaire

Amnionitis

Insuficiencia renal aguda

Septicemia

TOTALES
PLACENTA

PREVIA

\section{RUPTURA \\ D.P.P.N.I. UTERINA}

TOTAL

No. $\%$ No. $\%$ No. $\%$ No. $\%$

\begin{tabular}{|c|c|c|c|c|c|c|c|c|}
\hline C:I-D. & 0 & & 6 & 6.6 & 0 & & 6 & 31.5 \\
\hline Utero de Couvelaire & 0 & & 8 & 8.8 & 0 & & 8 & 42.2 \\
\hline Amnionitis & 1 & 1.1 & 0 & & 1 & 25 & 2 & 10.5 \\
\hline Insuficiencia renal aguda & 0 & & 1 & 1.1 & 0 & & 1 & 5.3 \\
\hline Septicemia & 0 & & 0 & & 2 & 50 & 2 & 10.5 \\
\hline TOTALES & 1 & & 15 & & 3 & & 19 & $10.2 \%$ \\
\hline
\end{tabular}

CUADRO No. 10

HEMORRAGIAS DE LA SEGUNDA MITAD DE LA GESTACION TRATAMIENTO REALIZADO

TIPO DE
TRATAMIENTO

Liquidos y electrolitos

Liquidos-corticoides

Liquidos-sangre

Liquidos-corticoides sangre

Liquidos-corticoides heparina

Liquidos-corticoides sangre-heparina

Liquidos-heparina ácido épsilon-aminocaproico

TOTALES
PLACENTA PREVIA
RUPTURA

D.P.P.N.I. UTERINA TOTAL

No. $\%$ No. $\%$ No. $\%$ No. $\%$

\begin{tabular}{|c|c|c|c|c|c|c|c|}
\hline $\begin{array}{r}83 \\
0\end{array}$ & 91.2 & $\begin{array}{l}28 \\
10\end{array}$ & $\begin{array}{l}30.8 \\
11\end{array}$ & $\begin{array}{l}0 \\
0\end{array}$ & & $\begin{array}{l}111 \\
10\end{array}$ & $\begin{array}{r}60.2 \\
5.5\end{array}$ \\
\hline 8 & 8.8 & 17 & 18.6 & 1 & 25 & 26 & 13 \\
\hline 0 & & 11 & 12.1 & 3 & 75 & 14 & 7.5 \\
\hline 0 & 16 & 16 & 17.6 & 0 & & 16 & 9 \\
\hline 0 & & 8 & 8.8 & 0 & & 8 & 4.4 \\
\hline
\end{tabular}

$\begin{array}{lllllll}0 & 1 & 1 & 1.1 & 0 & 1 & 0.6\end{array}$

$\begin{array}{llllllll}91 & 100 & 91 & 100 & 4 & 100 & 186 & 100 \%\end{array}$ 


\section{CUADRO No. 11}

\section{HEMORRAGIAS DE LA SEGUNDA MITAD DELA GESTACION OTRAS CIRUGIAS REALIZADAS}

\section{CIRUGIAS}

SALPINGOTOMIAS

HISTEREC- Subtotal TOMIA

Total

PLACENTA PREVIA
RUPTURA D.P.P.N.I. UTERINA No. $\%$ No. $\%$ 17 17 18.7

$$
25
$$$$
25
$$$$
27.5
$$$$
0
$$$$
9.9
$$$$
4
$$$$
100
$$$$
\begin{array}{ll}
42 & 73.7
\end{array}
$$

0

$2 \quad 2.2 \quad 0$

\section{CUADRO No. 12}

HEMORRAGIAS DE LA SEGUNDA MITAD DE LA GESTACION RELACION FETO-MATERNA DE MORTALIDAD

\begin{tabular}{|c|c|c|c|c|c|c|}
\hline \multirow{3}{*}{ ENTIDADES } & \multicolumn{3}{|c|}{ FETOS } & \multicolumn{3}{|c|}{ MADRES } \\
\hline & \multirow{2}{*}{$\begin{array}{c}\text { VIVOS } \\
\text { No. }\end{array}$} & \multicolumn{2}{|c|}{ MUERTOS } & \multirow{2}{*}{$\begin{array}{c}\text { VIV AS } \\
\text { No. }\end{array}$} & \multicolumn{2}{|c|}{ MUERTAS } \\
\hline & & No. & $\%$ & & No. & $\%$ \\
\hline $\begin{array}{l}\text { PLACENTA } \\
\text { PREVIA }\end{array}$ & 82 & $\begin{array}{c}9 \\
\left(15.8^{\mathrm{o}} / \mathrm{o}\right)\end{array}$ & 9.9 & 91 & 0 & 0 \\
\hline D.P.P.N.I. & 47 & $(77.2 \%$ & 48.4 & 83 & $(4.3 \%$ & 8.8 \\
\hline $\begin{array}{l}\text { RUPTURA } \\
\text { UTERINA }\end{array}$ & $\begin{array}{l}0 \\
0\end{array}$ & $\begin{array}{c}4 \\
(7 \%)\end{array}$ & 100 & 4 & 0 & 0 \\
\hline TOTALES & $\begin{array}{l}129 \\
(69.4 \%)\end{array}$ & 57 & $30.6 \%$ & 178 & 8 & $4.3 \%$ \\
\hline
\end{tabular}


CUADRO No. 13

HEMORRAGIAS DE LA SEGUNDA MITAD DE LA GESTACION RELA CION FETAL

\begin{tabular}{|c|c|c|c|c|c|c|c|c|}
\hline \multirow{3}{*}{$\begin{array}{l}\text { FETOS } \\
\text { ž } \\
\text { ô Normales }\end{array}$} & \multicolumn{2}{|c|}{$\begin{array}{l}\text { PLACEINTA } \\
\text { PREVIA }\end{array}$} & \multicolumn{2}{|c|}{ D.P.P.N.I. } & \multicolumn{2}{|c|}{$\begin{array}{c}\text { RUPTURA } \\
\text { UTERINA }\end{array}$} & \multicolumn{2}{|c|}{ TOTAL } \\
\hline & No. & $\%$ & No. & $\mathrm{o}_{\mathrm{o}}$ & No. & $\%$ & No. & $\%$ \\
\hline & 82 & 90.1 & 39 & 42.8 & 1 & 25 & 122 & 65.6 \\
\hline Ŝf Sufrimiento & 3 & 3.3 & 16 & 17.6 & 0 & & 19 & 10.3 \\
\hline Muertos & 4 & 4.4 & 28. & 30.8 & 3 & 75 & 35 & 18.7 \\
\hline Sin datos & 2 & 2.2 & 8 & 8.8 & 0 & & 10 & 5.4 \\
\hline Normales & 69 & 75.8 & 31 & 34 & 0 & & 100 & 53.8 \\
\hline Deprimidos & 13 & 14.3 & 16 & 17.6 & 0 & & 29 & 15.6 \\
\hline$\widetilde{\varepsilon}$ Muertos & 9 & 9.9 & 44 & 48.4 & 4 & 100 & 57 & 30.6 \\
\hline TOTALES & 91 & & 91 & & 4 & & 186 & $100 \%$ \\
\hline
\end{tabular}

CUADRO No. 14

HEMORR AGIAS DE LA SEGUNDA MITAD DE LA GESTACION DEFUNCION FETAL

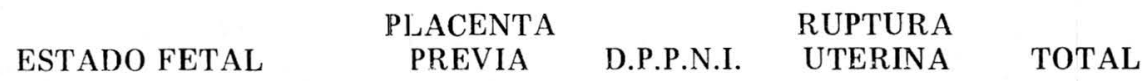

No. $\%$

$\begin{array}{lllllll}\text { AL } & \text { Normales } & 0 & 0 & 1 & 1 & 1.8\end{array}$

INGRESO

$\begin{array}{cllllcc} & \begin{array}{c}\text { con } \\ \text { sufrimien- } \\ \text { to } \\ \text { Muertos }\end{array} & 3 & 16 & 0 & 19 & 33.3 \\ & \begin{array}{c}\text { sin } \\ \text { datos }\end{array} & 2 & 28 & 3 & 35 & 61.4 \\ & \text { Muertos } & 9 & 0 & 0 & 2 & 3.5 \\ & & (15.8 \%) & (77.2 \%) & (4 \%) & (30.6 \%)\end{array}$




\section{CUADRO No. 15}

HEMORR AGIAS DE LA SEGUNDA MITAD DE LA GESTACION GRUPO SANGUINEO Y FACTOR Rh. MATERNO

GRUPO DE SANGRE FACTOR Rh.

$0+$
$0-$
$\mathrm{A}+$
$\mathrm{A}-$
$\mathrm{B}+$
$\mathrm{B}-$
$\mathrm{AB}+$
$\mathrm{AB}$.

TOTALES
PLACENTA PREVIA

\section{RUPTURA \\ D.P.P.N.I. UTERINA}

TOTAL No. $\%$ 74 2 21 3 15 3 4 2
2

$52(57 \%)$

$69(75.8 \%) \quad 3(75 \%)$ $124(66.7 \%)$

\section{CUADRO No. 16}

HEMORRAGIAS DE LA SEGUNDA MITAD DE LA GESTACION DEFUNCION MATERNA

No.

T R A T A M I E N T O

D.P.P.N.I.

COMPLICACIONES Casos sangre heparina C.S.T. vaginal tomia No. $\%$

C.I.D.

I. R. A.

ECLAMPSIA

TOTAL

$\begin{array}{ll}4 & 4 \\ 3 & 3 \\ 1 & 1 \\ 8 & 8\end{array}$

2

3

21

1

$4 \quad 50$

2

1

$3 \quad 37.5$

1

-

1

$\begin{array}{lll}1 & 12.5\end{array}$

6

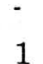

$8 \quad 4.3 \%$ 


\section{CUADRO No. 17}

\section{HEMORR AGIAS DE ILA SEGUNDA MITAD DE LA GESTACION PORCENTAJE DE .MORTALIDAD FETAL EN EL D.P.P.N.I. \\ SEGUN AUTORES}

\section{AUTORES}

SCHWARCZ. R.

HELLMAN. L. (WILLIAMS)

GREENHILL. J. P.

MCLENNAN. CH.

LA NUESTRA в Blanco-Martínez y Còl(presente trabajo)

\section{CUADRO No. 18}

HEMORRAGIAS DE LA SEGUNDA MITAD DE LA GESTACION PORCENTAJE DE MORTALIDAD MATERNA EN EL D.P.P.N.I.

\section{SEGUN AUTORES}

AUTORES

HELLMAN. L.

MCLENNAN. CH.

GREENHILL. J. P.

SCHW ARCZ. R.

LA NUESTRA B Blanco-Martínez y Coí(presente trabajo)

\section{CUADRO No. 19}

\section{HEMORRAGIAS DE LA SEGUNDA MITAD DEL EMBARAZO}

\section{NORMAS TERAPEUTICAS}

I. HISTORIA CLINICA (Anamenesis y exámen físico)

II- CANALIZAR VENA

III- MUESTRA DE SANGRE PARA:
a. Cuadro hemático.
b. Hemoclasificar y cruzar.
c. Tiempo de coagulación y sangría.
d. Tiempo de Protrombina
e. Recuento de Plaquetas.
f. Observación del coágulo 


\section{CUADRO No. 20}

\section{HEMORRAGIAS DE LA SEGUNDA MITAD DEL EMBARAZO}

\section{MANEJO TERAPEUTICO}

1. Líquidos y Electroliticos

2. Sangre total.

3. Corticoides.

4. Antibioticos.

5. Heparina.

6. Tratamiento obstétrico adecuado y oportuno.

7. Médidas especiales.

8. Control del Recien Nacido

\section{RESUMEN}

En el Departamento de Obstetricia y Ginecología de la Facultad de Medicina de Cartagena, se estudiaron 186 casos de hemorragias de la segunda mitad de la gestación. Los casos fueron seleccionados de 25.286 Historias Clínicas, correspondientes a los años 1976, 1977 y 1978, de la Clínica de Maternidad "Rafael Calvo C. y el Hospital Universitario de Cartagena. Anotamos que aproximadamente 9 de cada 1000 embarazadas, en la segunda mitad de la gestación, padecen hemorragias.

Clasificadas estas hemorragias según entidades, resultaron para los 3 años, 91 pacientes con Placenta Previa, el mismo número con D.P.P.N.I. y 4 Rupturas Uterinas.

La edad promedio de las pacientes con Placenta Previa fue de 29.1 años; para las de D.P.P.N.I. fue de 29.6 .

La paridad promedio para la Placenta Previa fue de 3.8 partos, para el D.P.P.N.I. fue de 4 . En las nulíparas el D.P.P.N.I'* es 3 veces más frecuente que la Placenta Previa.

* Desprendimiento de placenta normo-incerta
La Placenta Previa y el D.P.P.N.I. se presentaron con marcada frecuencia desde las 34 y más semanas de embarazo. Los casos de Rupturas Uterinas, todos, fueron en embarazos a término.

El motivo de consulta predominante en la Placenta Previa, fue el sangrado indoloro $(81.3 \%)$. El sangrado con dolor predominó en el D.P.P.N.I. (59.3\%) y el dolor en la Ruptura Uterina (75\%).

La hipertonía uterina - estuvo presente en el D.P.P.N.I., con $42 \%$. La situación trasversa del feto, la cesárea anterior y el manejo por empírica se asociaron con la rup tura uterina en $75 \%$

Las complicaciones maternas predomina ron en el D.P.P.N.I., con $79 \%$ Sobresaler el Utero de Couvelaire (42.2\%) y la C.I.D (13.5\%).

El tipo de parto que predominó, fue la Ce sárea segmentaria transperitoneal, con 84 $9 \%^{-}$y fue seguido por el parto vaginal espon táneo con $10.7 \%$ El $60 \%$ de pacientes fu manejado solamente con líquidos y electro litos; además de otras medidas generales y especiales. El $25 \%$ recibió corticoides y sangre total. El uso de la Heparina fue ex 
clusivo para el D.P.P.N.I. en 25 casos (27. $5 \%)$. De éstos, 21 (84\%) evolucionario satisfactoriamente y 4 (16\%) murieron.

La mortalidad fetal fue de 57 casos ( 30. $6 \%$ ). De éste, corresponde a la placenta previa $4.9 \%$ al D.P.P.N.I., $23.5 \%$ y a la Ruptura Uterina, $2.2 \%$ Analizada esta mortalidad separadamente para ca.da entidad, según número de casos, corresponde a Placenta Previa $9.9 \%$ al D.P.P.N.I., $48.4 \%$ y a la Ruptura Uterina $100 \%$

El $61^{\circ} / 0$ de los fetos fueron mortinatos desde el ingreso $; 33.3^{\circ} /$ tenía sufrimiento y $1.8 \%$ estaba normal.

La mortalidad materna exclusiva del D.P.P. N.I. fue de 8 casos $(8.8 \%)$. Para el número total de casos (186) esta mortalidad, corresponde a $4.3 \%$

\section{SUN MARY}

186 cases of hemor hage in the second half of gestation were studied in the Obstetrics and Gynecology Department of Cartagena's School of Medicine. The cases were chosen from 25.286 Clinical Reccrds corresponding to 1976, 1977 and 1978 of Maternity Hospital "Rafael Calvo C." and Cartagena's Hospital Universitario. We noted that aproximately 9 of each 1000 pregnant women suffered hemorrhages during the second half of gestation.

The results for the three years, after classifying these hemorrhage according to entities, were: 91 patients previous Placenta, the same number with P.P.D.N.I.P. (Premature Placenta Detarchment in NormoInserted Placenta) and 4 Ruptures of the Uterus.

The average age of the patients with Previous Placenta was 29.1 years; for patients with P.P.D.N.I.P. was 29.6.

The average parity in Previous Placenta was 3.8 deliveries and in P.P.D.N.I.P. 4. P.P.D.N.I.P. is three times more frequent in multiparas than Previous Placenta.

Previous Placenta and P.P.D.N.I.P. appeared more frequently after more than 34 weeks of pregnancy. All cases of Ruptures of the Uterus appeared in almost completed pregnancies.

The outstanding reason for medical examination in Previous Placenta was pinless bleeding $(81.3 \%)$. Bleeding with pain prevailed in the P.P.D.N.I.P. (59.3\%) and pain in the Rupture of the Uterus $(75 \%)$.

$42 \%$ of the cases with P.P.D.N.I.P. had Uterine Hypertonia. The cross possition of the fetus, the anterior Caesarean operation and empiricism were related to the Rupture of the Uterus in $75 \%$ of the cases.

Maternal complications prevailed in P.P.D. N.I.P. with $79 \%$. The most outstanding cases are Couvalaire Uterus $(42.2 \%)$ and C.I.D. (13.5\%).

The prevailing delivery was transperitoneal segmentary Caesarea with $84.9 \%$, followed by spontaneous vaginal delivery with $10.7 \% .60 \%$ of the patients were only given fluids and electrolites besides other general and special meassures. $25 \%$ received corticoides and total blood. The use of heparine was exclusively for P.P.D.N.I.P. in 25 of the cases $(27.5 \%)$, of which 21 (84\%) developed satisfactorily and $4(16 \%)$ died.

Fetal mortality arouse in 57 cases (30. $6 \%$ ), divided as follows: $4.9 \%$ correspond to Previous Placenta, 23.5\% toP.P.D.N.I.P. and $2.2 \%$ to Rupture of the Uterus. After analizing this mortality rate separately for each entity and according to the number of cases, $9.9 \%$ correspond to Previous Placenta, $48.4 \%$ to P.P.D.N.I.P. and $100 \%$ to Rupture of the riterus.

$61 \%$ of the fetus born with Apgar Cero were dead at the time of the admission; $33.3 \%$ were suffering and $1.8 \%$ were normal.

The maternal mortality exclusive of P.P.D. 
N.I.P. appeared in 8 cases $(8.8 \%)$. Form the total number of cases (186) this mortality represents $4.3 \%$.

\section{RECOMENDACIONES}

Nuestra -pequeña - experiencia, nos deja la impresión de, que para reducir la mortalidad fetal y materna en el Desprendimiento Prematuro de Placenta Normoinserta, es necesario:,

1. Ser más agresivo desde el punto de vista quirúrgico, ésto es, intervenir quirúrgicamente, cuanto antes llegue el caso a nuestras manos.

2. Dejar los partos vaginales, para aquellos casos en que éste sea inminente.

3. Hacer campañas en el sentido de que todos estos casos, cuanto antes lleguen al Hospital, su pronóstico será mejor, pues el tiempo transcurrido desde la iniciación del cuadro y el vaciamiento uterino, es directamente proporcional a la mortalidad fetal y materna.

\section{BII LIOGRAFIA}

1. BONNAR, J. "Coagulación de la sangre $y_{4}$ sistema fibrinolítico durante el embarazo". Temas actuales Gin. y Obst. Edit. Inter-americana, 319-338.1975.

2. BRUNO, R. "Fisiología del aborto". Ginecología Panamericana.Vol.2, 9-58.1971.
3. GOTTESFELD, K. "Ultrasonido en Obstetricia". Clínicas Obstétrica y Ginecológica, Edit. Inter-americana. Vol. 2.331.1978.

4. GREENHILL, L. FRIEDMAN, E. "Placenta previa, Desprendimiento de Placenta, Roturas Uterinas". Obstetricia. Primera Edición español. 409-420,657.1977.

5. HELLMAN, L. PRITCHARD, J. "Placenta previa y Abruptio Placentae". Obstetricia, Primera Edición. 530. 1973.

6. KARIM,R. CHEVLI,R. "Hemorragia y choque pre-parto" Clínicas Obst. y Ginecológicas, 533. sept. 1976.

7. KASER, O. "Hemorragias antes del parto". Gin. y Obst. Edit. Salvat. 743.1976.

8. LEVITT, M. ALTCHEK, A. “Hipertensión y toxemia gravídica". "Complicaciones Médicoquirúrgicas en el embarazo". Edit. Inter-americana. Segunda edición. 65. 1967.

9. LOUKA, M.H.; LEWIS, G.C. "Hemorragias obstétricas y ginecológicas". Tribuna Médica No. 690.11, agosto-1978.

10. MESSER, R. "Problemas de coagulación en Obstetricia". Clínicas Médicas de Norteamérica. 1085 , sep. 1969 .

11. MCLENNAN, CH. "Desprendimiento prematuro de placenta y placenta previa. Compendio de Obstetricia. Novena edición, 242. 1977.

12. RICE, R. SERRANO, C. "Mortalidad neonatal por afecciones de la placenta y el cordón umbilical" características de mortalidad en la niñez, 113-1973.

13. SANCHEZ, F. "Muerte fetal Intraparto". Rev. Colombiana de Ubstetricia y Ginecologia". 62.1978 .

14. SCHWARCZ, R. "Hemorragias de la segunda mitad del embarazo". Obstetricia. Tercera edición. Edit. El Ateneo, 519.1978. 